\title{
Current Trends of Dermatophytosis in Eastern Odisha
}

\author{
Swati Jain ${ }^{1}$ Sunita Kabi ${ }^{1}$ Bichitrananda Swain² \\ ${ }^{1}$ Department of Microbiology, Institute of Medical Sciences and \\ SUM Hospital, Bhubaneswar, Odisha, India \\ ${ }^{2}$ Department of Microbiology, SLN Medical College and Hospital, \\ Koraput, Odisha, India \\ J Lab Physicians:2020;12:10-14
}

\begin{abstract}
Address for correspondence Sunita Kabi, MBBS, MD, \#144, Sri Satyasai Enclave, Kolathia, Bhubaneswar, Odisha 751020, India (e-mail: sunitakabi@gmail.com).
\end{abstract}

\begin{abstract}
Keywords

- dermatophytes

- Epidermophyton

- Microsporum

- Trichophyton
\end{abstract}

Background Dermatophytes are fungi parasitizing structures rich in keratin such as skin, hair, and nail, which leads to a dermal inflammatory response with intense itching and cosmetic disfigurement. The climatic condition of eastern India is predominantly hot and humid with severe rainfalls promoting fungal infection. This study was performed to determine the prevalence of dermatophytosis and to isolate and identify the dermatophyte from clinically suspected cases attending tertiary care center.

Methodology This study was conducted from April 2018 to March 2019 and a total of 1,200 samples were included. Skin scrapings were collected and examined microscopically using $10 \%$ potassium hydroxide $(\mathrm{KOH})$ mount. Culture was performed using Sabouraud dextrose agar and dermatophyte test medium. Speciation was done based on macroscopic and microscopic findings using lactophenol cotton blue staining and some special tests like urease test and slide culture.

Results Of 1,200 cases, tinea corporis was the most common clinical presentation. Direct $\mathrm{KOH}$ positivity was $89.4 \%$ and culture positivity was $61.75 \%$. Trichophyton mentagrophyte $(77.5 \%)$ was the most common dermatophyte isolated followed by Trichophyton rubrum (13.3\%).

Conclusion Dermatophytic infections are not serious but their propensity to persist leading to chronicity and disfigurement may have personal and social implications.

\section{Introduction}

Dermatophytosis, commonly referred as "tinea" or "ringworm," has always been one of the most common superficial cutaneous mycotic infections encountered in the dermatology outpatient department. ${ }^{1}$ These are caused by a group of closely related keratinophilic fungi called dermatophytes, all of which produce enzyme keratinase and thus invade keratinized tissues of skin and its appendages like hair and nail. There are three genera of dermatophytes-Trichophyton, Microsporum, and Epidermophyton. ${ }^{2}$

Dermatophytosis is prevalent globally, affecting approximately 20 to $25 \%$ of the world's population. ${ }^{3}$ The prevalence is governed by environmental conditions, socioeconomic conditions, and individual's susceptibility. They are common in tropics like India due to factors such as heat, higher humidity, poverty, overcrowding, and poor hygienic living conditions. ${ }^{4,5}$
Several studies have been conducted in different parts of the country but the availability of scanty data on the prevalence of dermatophytosis in eastern India prompted us to take up the present study. We here present the largest crosssectional study from our region to determine the clinical pattern of dermatophytosis prevalent in our center, to isolate and speciate the dermatophytes.

\section{Materials and Methods}

This study was undertaken in the Department of Microbiology for a period of 1 year from April 2018 to March 2019 in IMS and SUM Hospital, Odisha. The specimens from all clinically suspected cases of dermatophytosis were subjected to mycological work-up. Cases of hair and nail infection and patients already taking antifungal treatment were excluded from the study.
License terms

() (1) $\ominus \circledast$ 


\section{Sample Collection}

After selection of an appropriate site, the affected area was cleaned with $70 \%$ ethanol and allowed to dry before collecting the specimen. Skin scrapings were obtained from the active edge of the lesion with the blunt end of a sterile surgical blade (number-15) held at an angle of 90 degrees. Samples were collected on sterilized white paper envelope. Each specimen was divided into two parts, one for direct microscopy and the other for fungal culture.

\section{Direct Microscopy}

Scrapings were mounted in fresh $10 \% \mathrm{KOH}$ on a clean grease free glass slide and observed under $10 \times$ and $40 \times$ magnifications for the presence of fungal filaments and arthrospores.

\section{Fungal Culture}

Samples were inoculated into dermatophyte test medium (DTM) and two Sabouraud dextrose agar (SDA) slopes, one with chloramphenicol (50 mg/L) + cycloheximide $(500 \mathrm{mg} / \mathrm{L})$ and the other with only chloramphenicol ( $50 \mathrm{mg} / \mathrm{L})$. The tubes were incubated in BOD (biochemical oxygen demand) incubator at 20 to $25^{\circ} \mathrm{C}$. Cultures were examined every alternate day for growth and were declared negative if no growth was observed till 4 weeks. Pure growth of isolate was obtained by subculturing on corn meal agar.

\section{Identification of Fungus}

The growth on SDA slope was examined for its topography (margin, folding, elevation), texture (cottony, woolly, velvety, powdery, granular) and pigmentation on the surface and reverse. DTM was used for presumptive identification of dermatophytes. Speciation was done using lactophenol cotton blue staining (LPCB) mount, cellophane tape mount, urease test, and slide culture whenever necessary. Characteristics of microconidia and macroconidia on LPCB mount help in the identification of different genera and species (-Figs. 1-4).
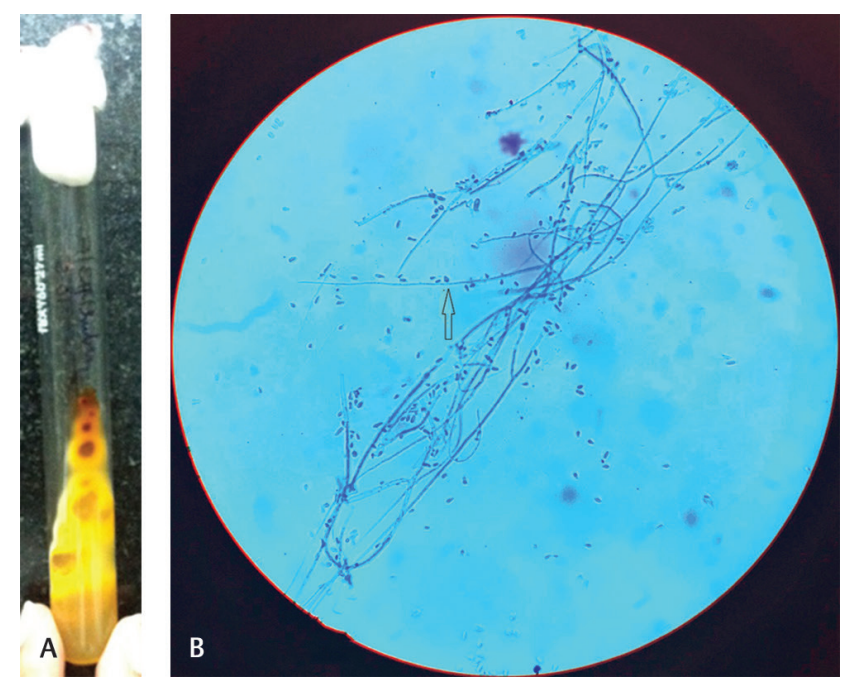

Fig. 1 Trichophyton rubrum: (a) SDA-wine red pigmentation on reverse side.(b) LPCB mount: arrowshowing teardrop-shaped microconidia. LPCB, lactophenol cotton blue staining; SDA, Sabouraud dextrose agar.
The obtained data from the study were analyzed by chisquare test using SPSS software.

\section{Results}

A total of 1,200 clinically suspected cases of dermatophytosis were included in this study.

Of 1,200 cases, the infection was seen to be more common among males (56.4\%) as compared with females (43.6\%) with a male:female ratio of $1.3: 1$. The infections were predominant in the age group of 21 to 40 years $(581 / 1,200)$, followed by $<20$ years $(346 / 1,200), 41$ to 60 years $(246 / 1,200)$, and least common in $>60$ years of age $(27 / 1,200)$. Maximum number of cases $(650 / 1,200)$ were seen between mid of summer and mid of monsoon (May-August).
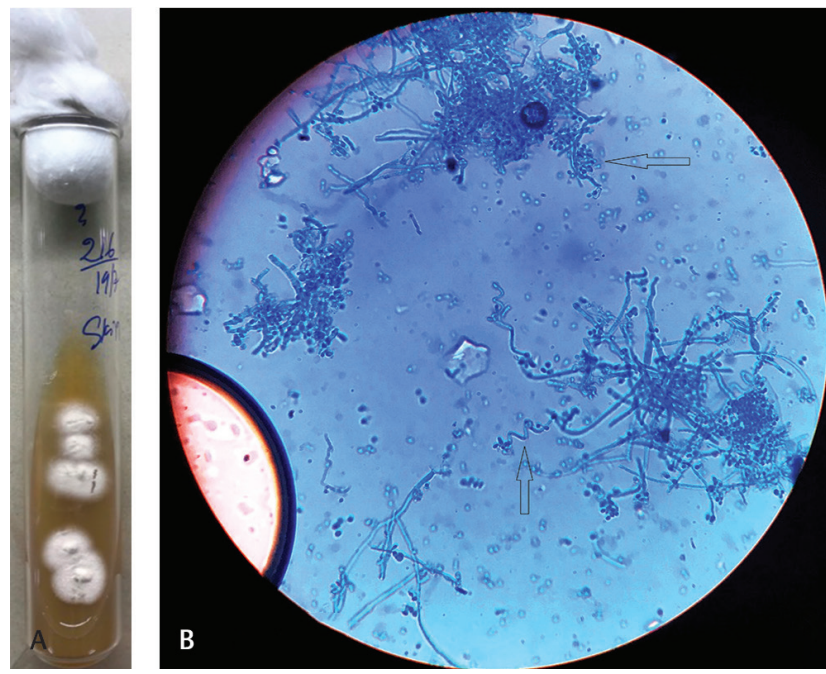

Fig. 2 Trichophyton mentagrophyte: (a) SDA-White colonies showing raised central tuft with granular surface. (b) LPCB mount: arrow showing spherical-shaped microconidia in clusters and spiral hyphae. LPCB, lactophenol cotton blue staining; SDA, Sabouraud dextrose agar.
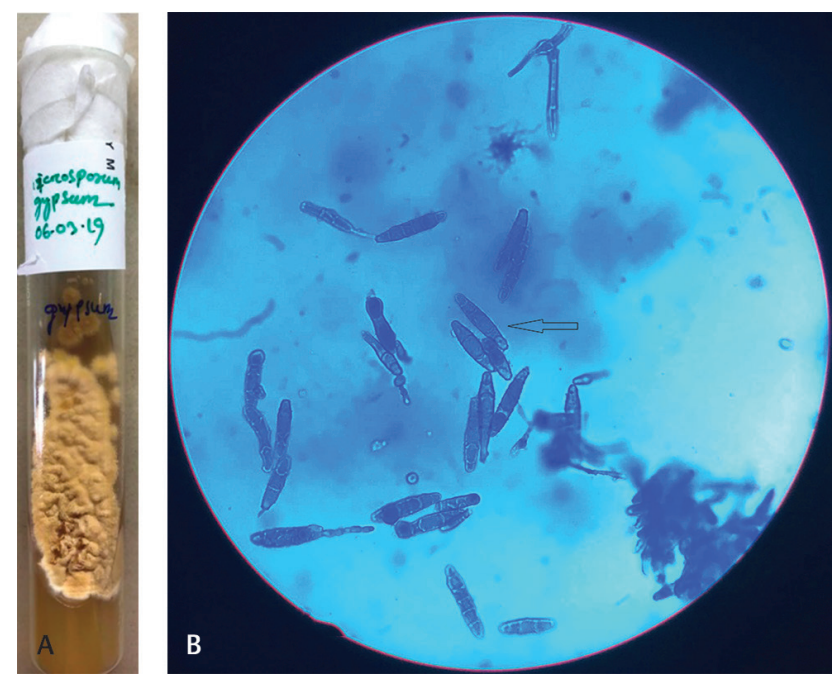

Fig. 3 Microsporumgypseum:(a)SDA-deepcream to buffcolored colonies with suede like surface.(b) LPCB mount: arrowshowing ellipsoidal-shaped, thin-walled, 4 to 6 celled macroconidia. LPCB, lactophenol cotton blue staining; SDA, Sabouraud dextrose agar. 

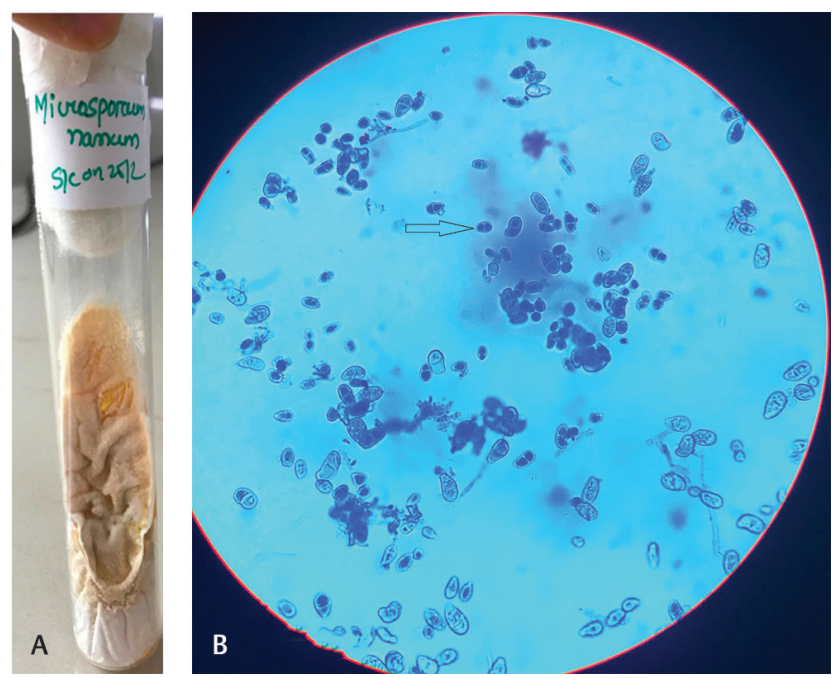

Fig.4 Microsporumnanum:(a)SDA-peach colored colonies with suedelike surface. (b) LPCB mount: arrow showing small ovoid- to pyriform-shaped, thin-walled, 1 to 3 celled macroconidia with broad truncate base. LPCB, lactophenol cotton blue staining; SDA, Sabouraud dextrose agar.

Table 1 Distribution of clinical presentations of dermatophytic infections

\begin{tabular}{|l|l|l|}
\hline Clinical presentation & No. of cases & Percentage \\
\hline Tinea corporis & 590 & $49.16 \%$ \\
\hline Tinea corporis + tinea cruris & 226 & $18.83 \%$ \\
\hline Tinea cruris & 193 & $16 \%$ \\
\hline Tinea faciei & 82 & $6.83 \%$ \\
\hline $\begin{array}{l}\text { Tinea cruris + tinea faciei + tinea } \\
\text { manuum }\end{array}$ & 65 & $5.41 \%$ \\
\hline Tinea pedis & 22 & $1.83 \%$ \\
\hline Tinea manuum & 13 & $1.1 \%$ \\
\hline Tinea incognito & 6 & $0.5 \%$ \\
\hline Tinea barbae & 3 & $0.25 \%$ \\
\hline
\end{tabular}

Table 2 Comparison of $\mathrm{KOH}$ mount with culture findings

\begin{tabular}{|l|l|l|l|}
\hline \multirow{2}{*}{ KOH mount } & \multicolumn{2}{|c|}{ Culture } & \multirow{2}{*}{ Total } \\
\cline { 2 - 3 } & Positive & Negative & \\
\hline Positive & 709 & 364 & 1,073 \\
\hline Negative & 32 & 95 & 127 \\
\hline Total & 741 & 459 & 1,200 \\
\hline
\end{tabular}

Among the clinical forms, the most common presentation was that of tinea corporis (49.16\%) followed by the combination of tinea corporis and tinea cruris (18.83\%) (- Table 1).

Of 1,200 suspected cases, 1,073 (89.4\%) were positive for fungal filaments by direct microscopy and 741 (61.75\%) were culture positive (-Table 2 ). The difference between positive and negative $\mathrm{KOH}$ mount for prediction of subsequent culture results was statistically significant with a value of 80.2464 $(p<0.05)$.

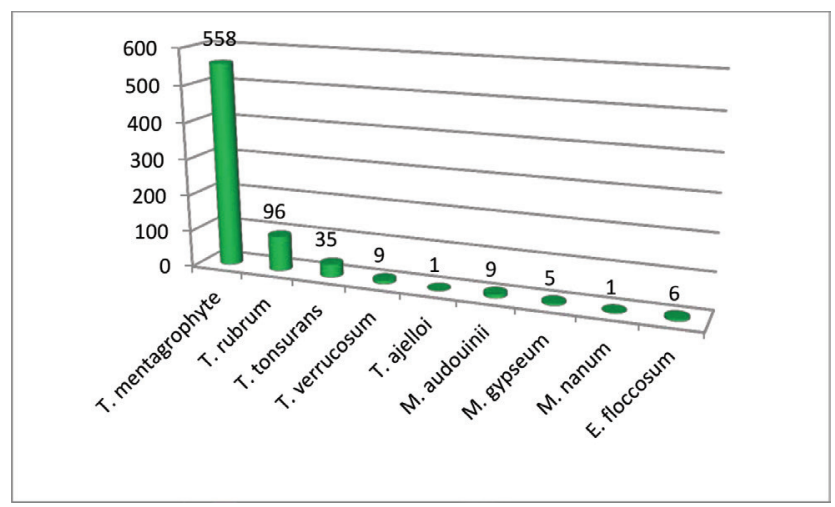

Fig. 5 Distribution of isolated dermatophytes.

Out of 741 culture positive cases, 720 were dermatophytes and 21 were yeasts. Yeasts were grown in the samples from tinea cruris, tinea pedis, and tinea faciei.

Of 720 dermatophytes isolated, Trichophyton species were implicated in 699 cases (97.08\%) while Microsporum and Epidermophyton species were isolated in 15 (2.08\%) and $6(0.83 \%)$ cases, respectively.

Trichophyton mentagrophyte was the predominant dermatophyte isolated (558/720) followed by Trichophyton rubrum (96/720) (-Fig. 5; - Table 3)

\section{Discussion}

Superficial fungal infections can be caused by dermatophytes as described above and nondermatophytes such as cutaneous candidiasis, pityriasis versicolor, tinea nigra, and black and white piedra. ${ }^{6}$

In the present study, 1,200 clinically suspected cases of dermatophytosis were included.

We have found male predominance in our study with a male: female ratio of 1.3:1. Similar finding has been reported by several researchers from different parts of India., ${ }^{17-9}$ This may be due to increased outdoor activity among males leading to increased perspiration thus facilitating fungal growth.

Our study suggests dermatophytic infection is common in the adult age group (21-40 years) which is well correlated with the other studies. ${ }^{7,9}$ This could be due to the working culture which predisposes them to climatic conditions. Also, socialization is high in this age group which eventually helps in spreading of infection.

Maximum number of cases $(650 / 1,200)$ were seen between mid of summer and mid of monsoon compared with other two quarters. This could be due to favorable temperature $\left(35-40^{\circ} \mathrm{C}\right)$ and humidity $(>85 \%)$ which provide a fertile ground for the growth of fungus.

In the current study, tinea corporis was the commonest clinical presentation followed by combination of tinea corporis and tinea cruris. This finding is well corroborated with several other studies. ${ }^{1,8,10}$

In our study, 1,073 cases (89.4\%) were positive by $\mathrm{KOH}$ mount, 741 cases $(61.75 \%)$ were positive by culture and 709 cases (59\%) were positive by both $\mathrm{KOH}$ and culture. This result is comparable with studies by Mishra et al and 


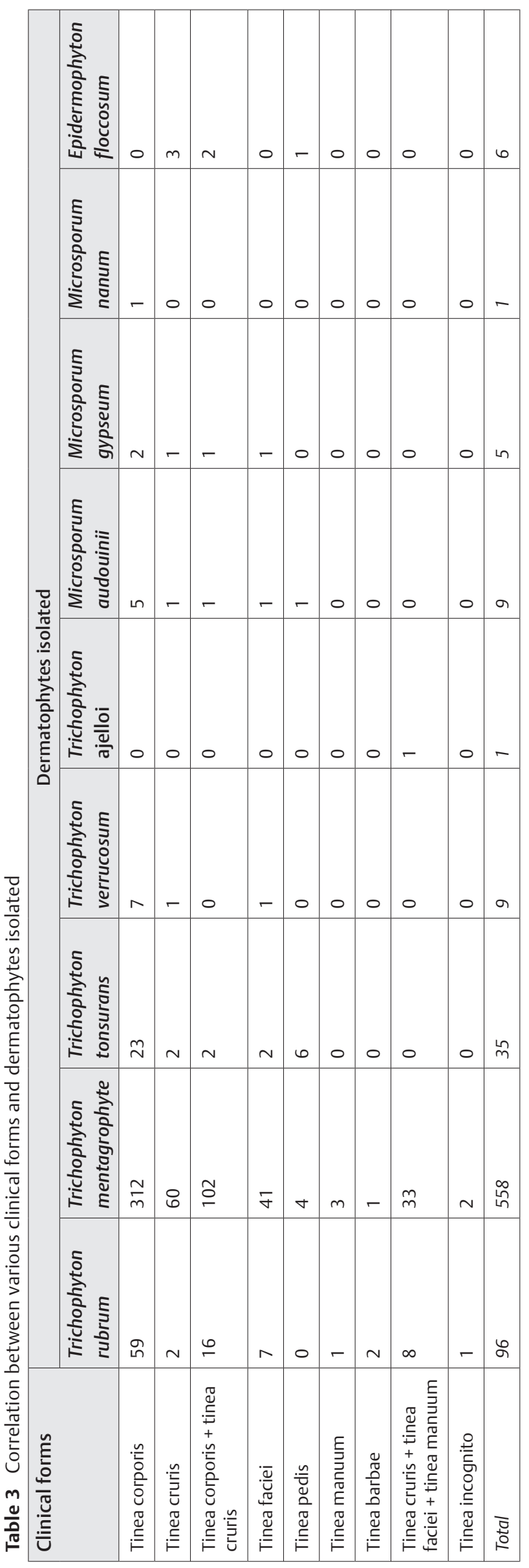

Jain et al where $\mathrm{KOH}$ positivity was 85 and $72 \%$ and culture positivity was 64 and $62 \%$, respectively. ${ }^{11,12}$ These studies show that both direct microscopy and culture play important role in the diagnosis of fungal infection.

Trichophyton species have been a major causative agent of dermatophytosis than the other two genuses, Microsporum and Epidermophyton. In our study also, among 720 dermatophytes isolates, genus Trichophyton was responsible for majority of cases (97.08\%) and only negligible number of isolates of Microsporum and Epidermophyton were grown, a finding which concurs with several other studies. ${ }^{1,9}$

T. mentagrophyte (77.5\%) was the most common dermatophyte isolated in our study followed by T. rubrum (13.3\%) which is similar to other studies.,13,14 This finding is contrary to the observation of other studies in which a reverse trend has been reported., ${ }^{4,5,8}$ T. rubrum is generally linked to chronic dermatophytosis and we do not have exact data about the chronic cases. Besides, the use of effective and prolonged fungal therapy for treatment might have reduced the incidence of T. rubrum in this region. Also, in recent years, incidence of T. mentagrophytes is increasing gradually.

\section{Conclusion}

Dermatophytoses are distributed worldwide with increased incidence in tropical countries like India. In spite of therapeutic advances in the last decades, the incidence is on the rise and we are facing an onslaught of dermatophytosis in volumes never encountered previously. Though clinical diagnosis of dermatophytosis is easy enough for an experienced dermatologist, still there are chances of misdiagnosis as many of these skin lesions may mimic other skin disorders. This may lead to unwarranted use of antifungal agents or steroids. Diagnosis of these fungal infections in the laboratory needs to be encouraged because of the ease with which these organisms can be identified with minimal basic infrastructure before initiating antifungal therapy. Despite the current upsurge of dermatophytosis in India, it probably does not get the attention it deserves as far as scientific research is concerned. Antifungal susceptibility studies should be performed as increased and prolonged use of antifungal agents has led to the development of resistance.

\section{Conflict of Interest}

None declared.

\section{References}

1 Bhatia VK, Sharma PC. Epidemiological studies on dermatophytosis in human patients in Himachal Pradesh, India. Springerplus 2014;3:134

2 Kaufman G, Berdicevsky I, Woodfolk JA, Horwitz BA. Markers for host-induced gene expression in Trichophyton dermatophytosis. Infect Immun 2005;73(10):6584-6590

3 Ménan EI, Zongo-Bonou O, Rouet F, et al. Tinea capitis in schoolchildren from lvory Coast (western Africa). A 1998-1999 cross-sectional study. Int J Dermatol 2002;41(4):204-207

4 Sharma S, Borthakur AK. A clinico-epidemiological study of dermatophytosis in north east India. Indian J Dermatol Venerol Leprol 2007;73:427-428 
5 Peerapur BV, Inamdar AC, Pushpa PV, Srikant B. Clinicomycological study of dermatophytosis in Bijapur. Indian J Med Microbiol 2004;22(4):273-274

6 Verma S, Heffernan MP, Superficial fungal infection: dermatophytosis, onychomycosis, tinea nigra, piedra. In: Wolff $\mathrm{K}$, Goldsmith LA, Katz SI, Gilchrest BA, Paller AS, Leffell DJ, eds. Fitzpatrick's Dermatology in General Medicine. Philadelphia, PA: McGraw Hill Professional; 2008 1807-1831

7 Maulingkar SV, Pinto MJ, Rodrigues S. A clinico-mycological study of dermatophytoses in Goa, India. Mycopathologia 2014;178(3-4):297-301

8 Balakumar S, Rajan S, Thirunalasundari T, Jeeva S. Epidemiology of dermatophytosis in and around Tiruchirapalli, Tamil Nadu, India. Asian Pac J Trop Dis 2012;2(4):286-289

9 Clarissa LJ, Valarie LW, Basabdatta C, Sangma KA, Bora I, Khyriem AB. Clinico-mycological profile of dermatophytosis in Meghalaya. Int J Med Public Health 2013;3:254-256
10 Ardeshna KP, Rohatgi S, Jerajani HR. Successful treatment of recurrent dermatophytosis with isotretinoin and itraconazole. Indian J Dermatol Venereol Leprol 2016;82(5):579-582

11 Sharma M, Sharma R, Singh PC, Mishra BC. Profile of dermatophytic and other fungal infections in Jaipur. Indian J Microbiol 2012;52(2):270-274

12 Jain N, Sharma M, Saxena VN. Clinico-mycological profile of dermatophytosis in Jaipur, Rajasthan. Indian J Dermatol Venereol Leprol 2008;74(3):274-275

13 Negi N, Tripathi V, Choudhury RC, Bist JS, Kumari N, Chandola I. Clinicomycological profile of superficial fungal infections caused by dermatophytes in a tertiary care centre of North India. Int J Curr Microbiol Appl Sci 2017;6(8):3220-3227

14 Sharma R, Adhikari L, Sharma RL. Recurrent dermatophytosis: a rising problem in Sikkim, a Himalayan state of India. Indian J Pathol Microbiol 2017;60(4):541-545 